\title{
ANOTHER PROOF OF BIANCHI'S IDENTITY IN RIEMANNIAN GEOMETRY
}

\author{
JERRY L. KAZDAN ${ }^{1}$
}

It is well known that the Riemann curvature tensor satisfies the two Bianchi identities (in standard tensor notation)

$$
\begin{aligned}
R_{i j k l}+R_{i l j k}+R_{i k l j} & =0, \\
R_{i j k, l}+R_{t i l j, k}+R_{t i k l, j} & =0 .
\end{aligned}
$$

These have always seemed a bit mysterious, despite their short proofs from an abstract viewpoint [KN, p. 121]. From the work of DeTurck [DT] on Ricci curvature, it became clear that the Bianchi identities are intimately related to the group of diffeomorphisms. This led to the following natural and conceptually transparent proof. The approach is basic to subsequent treatment of the curvature formulas as partial differential equations. Unfortunately, the computation has its unpleasant moments. It is evident that the same procedure can be applied elsewhere.

Given a metric $g$, let $\operatorname{Riem}(g)$ be its curvature tensor. Let $\phi_{\lambda}$ be a family of diffeomorphisms of $\mathbf{R}^{n}$ depending on a parameter $\lambda$, with $\phi_{0}=$ identity. Clearly

$$
\phi_{\lambda}^{*}(\operatorname{Riem}(g))=\operatorname{Riem}\left(\phi_{\lambda}^{*}(g)\right)
$$

for all $\lambda$. Take the derivative of (3) with respect to $\lambda$ and evaluate the result at $\lambda=0$. This gives an identity. This identity is equivalent to (1) and (2). So much for the idea. Now the tedious details.

Let $v^{l}$ be the vector field generating $\phi_{\lambda}$ so $\left.\left(d \phi_{\lambda} / d \lambda\right)^{l}\right|_{\lambda=0}=v^{l}$. Then for any $(1,3)$ tensor field, such as $R_{i j k}^{l}$, we have

$$
\begin{aligned}
{\left[\left.\frac{d}{d \lambda}\right|_{\lambda=0} \phi_{\lambda}^{*}(\operatorname{Riem}(g))\right]_{i j k}^{l}=} & v^{t} R_{i j k, t}^{\prime}+v_{, i}^{s} R_{s j k}^{\prime}+v_{, j}^{s} R_{i s k}^{\prime} \\
& +v_{, k}^{s} R_{i j s}^{\prime}-v_{,{ }_{,}}^{l} R^{s}{ }_{i j k}
\end{aligned}
$$

which is the left side of (3) after $\lambda$ differentiation.

For the right side of (3), one can easily verify that for any family of metrics $g_{\lambda}$ with $d g_{\lambda} / d \lambda=h$ at $\lambda=0$, the first variation of the curvature tensor is

$$
\left[\left.\frac{d}{d \lambda}\right|_{\lambda=0} \operatorname{Riem}\left(g_{\lambda}\right)\right]_{i j k}^{l}=\frac{1}{2} g^{l s}\left(h_{i s, k j}+h_{k s, i j}-h_{k i, s j}-h_{i s, j k}-h_{j s, i k}+h_{j i, s k}\right) \text {. }
$$

Received by the editors March 9, 1980.

1980 Mathematics Subject Classification. Primary 53B20; Secondary 53A45.

'Supported in part by NSF Grant \#MCS-7901780. 
In our case of (3), $g_{\lambda}=\phi_{\lambda}^{*}(g)$ so $h_{i j}=\left(v_{i, j}+v_{j, i}\right)$. Consequently (5) will contain third derivatives of $v$. However, using the Ricci commutation formulas, all the third and second derivatives of $v$ cancel, leaving

$$
\begin{aligned}
\left.\frac{d}{d \lambda}\right|_{\lambda=0} \operatorname{Riem}\left(\phi_{\lambda}^{*}(g)\right)_{i j k}^{l} & \\
=\frac{1}{2} g^{l s}\{ & {\left[v^{t}\left(R_{s k t i}-R_{s i k t}+R_{s t i k}\right)\right]_{, j} } \\
& \left.+\left[v^{t}\left(R_{s i j t}-R_{s t i j}-R_{s j t i}\right)\right]_{, k}-2 v_{s,}^{t} R_{t i j k}+2 v_{, i}^{t} R_{s t j k}\right\} .
\end{aligned}
$$

Let $B_{i j k l}$ denote the left side of (1) and rewrite (6) as

$$
\begin{aligned}
= & \frac{1}{2}\left(v^{t} B_{k t i}^{l}\right)_{, j}-\frac{1}{2}\left(v^{t} B^{l}{ }_{j t i}\right)_{, k}+v^{t}\left(R_{i j t, k}^{l}-R_{i k t, j}^{l}\right) \\
& +v^{t}{ }_{, k} R_{i j t}^{l}+v^{t}{ }_{, j} R^{l}{ }_{i t k}-v^{l}{ }_{, t} R^{t}{ }_{i j k}+v^{t}{ }_{, i} R^{l}{ }_{t j k} .
\end{aligned}
$$

From (3), we know that (4) and (7) are equal. The last four terms in both expressions cancel, leaving the identity

$$
v^{t}\left(R_{i j k, t}^{l}+R_{i k t, j}^{l}+R_{i t j, k}^{l}\right)=\frac{1}{2}\left(v^{t} B_{k t i}^{l}\right)_{, j}-\frac{1}{2}\left(v^{t} B^{l}{ }_{j t i}\right)_{, k}
$$

which must hold for all vector fields $v$. Picking a vector field that is zero at a point we conclude that $v^{t}{ }_{j} B^{l}{ }_{k t i}-v^{t}{ }_{, k} B_{j t i}^{l}=0$. Since $R_{l k j i}=-R_{l k i j}$ then $B_{l k j i}=-B_{l j k i}$. Picking $v^{t}{ }_{j}=\delta^{t}$ at our point thus gives $B_{k j i}^{l}=0$, i.e., Bianchi's First Identity (1). Since our point was arbitrary, (1) must hold everywhere. Using this in (8) we immediately obtain Bianchi's Second.

\section{REFERENCES}

[DT] Dennis M. DeTurck, Existence of metrics with prescribed Ricci curvature (to appear).

[KN] S. Kobayashi and K. Nomizu, Foundations of differential geometry. I, Wiley-Interscience, New York and London, 1963.

Department of Mathematics, University of Pennsylvania, Philadelphia, Pennsylvania 19104 